\title{
The monotonic rising and oscillating of capillary driven flow in circular cylindrical tubes
}

\author{
Bo-Hua SUN ${ }^{1}$ \\ ${ }^{1}$ School of Civil Engineering 83 Institute of Mechanics and Technology, \\ Xi'an University of Architecture and Technology, Xi'an 710055, China \\ http://imt.xauat.edu.cn \\ email: sunbohua@xauat.edu.cn
}

\begin{abstract}
Among the best-known capillarity phenomena is a capillary rise, the understanding of which is essential in fluidics. Some capillary flows rise monotonically whereas others oscillate, but until now no criteria have been formulated for this scenario. In this paper, the Levine's capillary rise modelling is computed numerically, then the critical radius of the capillary tube is formulated by using the dimensional method and data fitting for identification of exponent index. The phase space diagram of capillary velocity versus height is obtained for the first time and shows that the phase transition from oscillating to monotonic rising happens when the phase trajectory decreases exponentially to somewhere other than the "attractor." Two general Maple codes of the problem are provided as an essential part of this paper.
\end{abstract}

Keywords: capillary rise, dynamics, tube radius criteria, oscillation, monotonic rising

\section{INTRODUCTION}

As shown in Fig. 1, capillary rise is among the bestknown and most vivid illustrations of capillarity. Understanding the laws of capillarity is important in many industries [1-20].

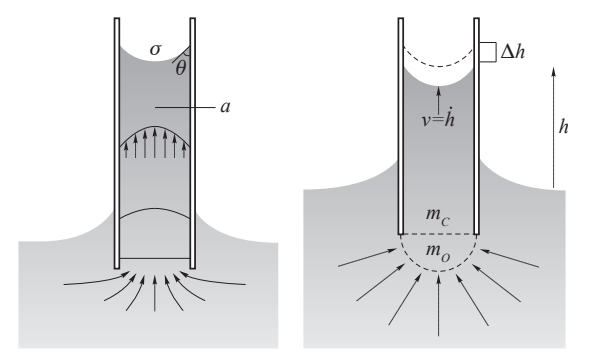

FIG. 1: The dynamics of capillary rise, where $\rho$ is density, $\mu$ is the viscosity, $\sigma$ is the liquid-air surface tension $\sigma=\gamma_{L V}, \theta$ is the wetting angle of the liquid, $h$ is the height of the capillary rise, $a$ is the capillary radius, and $g$ is the acceleration of gravity.

The physical dynamics can be briefly described as follows: Due to the effect of the surface tension, the capillary liquid obtains initial acceleration (the initial acceleration must never be zero), and begins to rise at a relatively uniform velocity, while the surface tension plays a dominant role in the ascending phase; however, as the capillary rises, wall frictions and gravity begin to work in an attempt to prevent the rise of the capillaries, and their joint action succeeds in decelerating the capillaries to a point, until the capillaries are finally stopped. Surface tension and wall resistance, as well as gravity to achieve unity of opposites, and the capillary dynamics process are attributed to calm.

The popular equation for capillary-rise dynamics is ob- tained as

$$
h \frac{d^{2} h}{d t^{2}}+\left(\frac{d h}{d t}\right)^{2}+\frac{8}{\rho a^{2}} \mu h \frac{d h}{d t}+g h=\frac{2}{\rho a} \sigma \cos \theta,
$$

which assumes a Poiseuille flow profile throughout the capillary. However, although this equation is used widely in the literature, it has a singularity at $t=0$, and more criticism of this equation can be found in $[7,11,15,19$, 20].

To remove the singularity, some scholars have reformulated the problem $[7,18]$. A singularity-free equation of capillary-rise dynamics has been obtained by Szekely, et al. [7] as follows:

$$
\left(h+\frac{7}{6} a\right) \frac{d^{2} h}{d t^{2}}+\frac{1}{2}\left(\frac{d h}{d t}\right)^{2}+\frac{8 \mu}{\rho a^{2}} h \frac{d h}{d t}+g h=\frac{2 \sigma}{\rho a} \cos \theta .
$$

Regarding the above Szekely's equation, Sun [19] proposed the capillary height as $h(t)=H\left[1-f(t) e^{-\beta t}\right]$, where $H=\frac{2 \sigma \cos \theta}{\rho g a}$. By Galerkin's method, he formulated a monotonic capillary height

$h(t)=\frac{2 \sigma \cos \theta}{\rho g a}\left\{1-\left[1+\left(\sqrt{\frac{6 g}{7 a}}\right) t+\alpha t^{3}\right] e^{-\left(\sqrt{\frac{6 g}{7 a}}\right) t}\right\}$,

as well as the capillary velocity

$$
\frac{d h(t)}{d t}=\frac{2 \sigma \cos \theta}{\rho g a}\left(\alpha t^{2} \sqrt{\frac{6 g}{7 a}}-3 \alpha t+\frac{6 g}{7 a}\right) t e^{-\left(\sqrt{\frac{6 g}{7 a}}\right) t} .
$$

However, Sun's approximate solution can not include capillary oscillation. In joint work with Zhong and Liao [20], we used homotopy analysis to attack the problem again and succeeded in finding both monotonic and oscillating solutions. The work by Zhong et al. [20] was the first attempt at simulating the entire process of capillary dynamics. 
After we have obtained the approximate analytical solution in [20], it is found that the solution is not practical due to its complicated structures. The reformulation will not be an easy task if changing capillary dynamics modelings. From an application point of view, it is highly demanded to have a simple and easy way of solving any capillary dynamics. Therefore, we will use commercial software to attack the problem. To make the programme coding as simple as possible, we use Maple to materialize the goal, because we found the Maple can provide us not only with the simplest programme but also having high accuracy.

Regarding the physics of capillary dynamics, there is a phenomena: monotonic rising and oscillation. However, there is no consensus on the criterion of the phase transition from oscillation to monotonic rising. It is still a open problem. This is the focus of our study.

In this paper, after introduction, in Section 2 we will convert Levine's nonlinear dynamics equation into state equations and provide a general Maple code for the problem. In Section 3 we apply the Maple code to study the influence of physical parameters on the capillary dynamics and confirm that the tube radius is a dominating parameter. In Section 4 we study the oscillation-monotonic phase transition problem by using stability analysis and propose a critical tube radius by dimensional analysis and data fitting. In Section 5 to find a depth mathematical criteria on the oscillation-monotonic phase transition, we study the phase state diagram. Finally with discussions and conclusion.

\section{LEVINE'S CAPILLARY DYNAMICS MODEL AND MAPLE CODE OF NUMERICAL SOLUTION}

According Stange et al. [12], one of the most detailed capillary theory up to now is proposed by Levine et al. [8]. In this paper, we will use Levine's model rather than Szekely's as we have used before. Levine's nonlinear dynamics equation is given as follows

$\left(h+\frac{37}{36} a\right) \frac{d^{2} h}{d t^{2}}+\frac{7}{6}\left(\frac{d h}{d t}\right)^{2}+\frac{\mu}{\rho}\left(\frac{8 h}{a^{2}}+\frac{2}{a}\right) \frac{d h}{d t}+g h=\frac{2 \sigma}{\rho a} \cos \theta$,

with initial height and velocity boundary conditions of $h(0)=0, \dot{h}(0)=0$.

For a comparative study, throughout the entire numerical investigation we use the data for diethyl ether in glass as given in Table I.

TABLE I: The diethyl ether in glass with arbitrary radius

\begin{tabular}{ccccc}
\hline \hline$\mu\left[\frac{\mathrm{kg}}{\mathrm{ms}}\right]$ & $\sigma\left[\frac{\mathrm{kg}}{\mathrm{s}^{2}}\right]$ & $\theta$ & $g\left[\frac{\mathrm{m}}{\mathrm{s}^{2}}\right]$ & $\rho\left[\frac{\mathrm{kg}}{\mathrm{m}^{3}}\right]$ \\
\hline $2.2 \cdot 10^{-4}$ & $1.67 \cdot 10^{-2}$ & $26^{0}$ & 9.81 & 710 \\
\hline \hline
\end{tabular}

Equation (2) is strongly nonlinear, therefore its solution cannot be obtained analytically and so must be computed numerically. The state equations of Eq. (2) are

$$
\begin{aligned}
& \frac{d h}{d t}=y, \\
& \frac{d y}{d t}=\frac{1}{h+\frac{37}{36} a}\left(\frac{2 \sigma}{\rho a} \cos \theta-\frac{7}{6} y^{2}-\frac{\mu}{\rho}\left(\frac{8 h}{a^{2}}+\frac{2}{a}\right) y-g h\right)
\end{aligned}
$$

and the initial conditions become $h(0)=0, y(0)=0$.

Although no analytical solution can be found, a numerical solution can be obtained. All the numerical simulations reported herein were done using the state equations Eq.3 and 4. To visualize and quantify the capillary dynamics process more precisely, we carry out comprehensive numerical investigations by Maple [21].

For easy use of results presented in this paper, the Maple code of computing the state equation with initial condition $h(0)=y(0)=0$ is provided below:

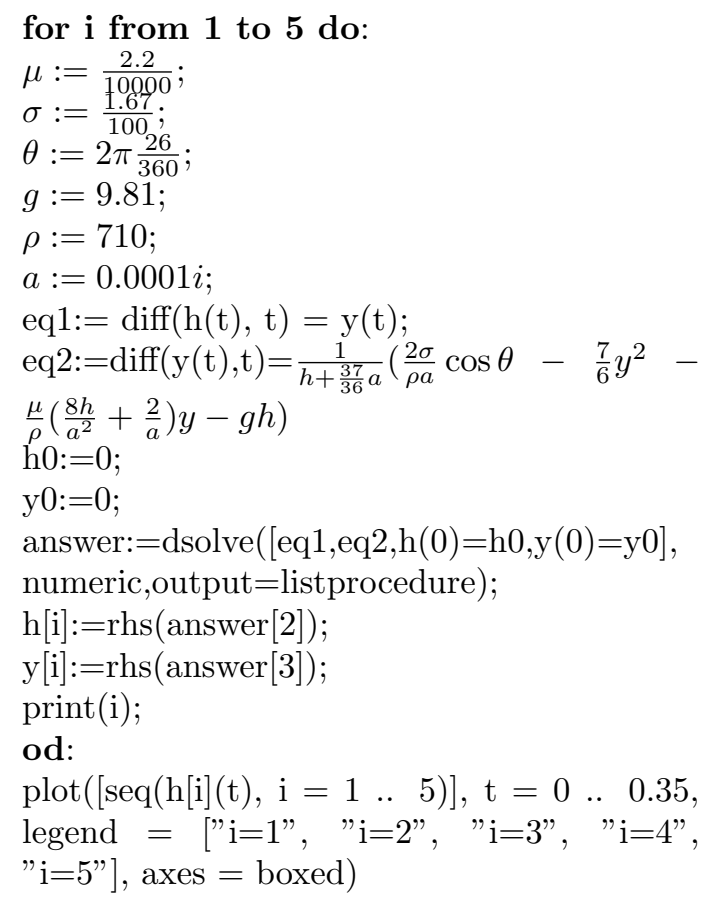

The code is general and can be used for other mathematical model by slightly modification. For comparison studies in the Section 3, we have also written a Maple code for Szekely's equation in Eq. 1, the Maple code is provided in the appendix. In the same way, Maple code of Stange's equation [12] can be easily done so we will not provide here.

\section{NUMERICAL CASE STUDIES}

Figure 2 compares the Jurin height $H=\frac{2 \sigma}{\rho g a} \cos \theta$ and Sun's solution [19], showing that the latter is simply an 
average of the real solution and does not capture the oscillatory behavior.

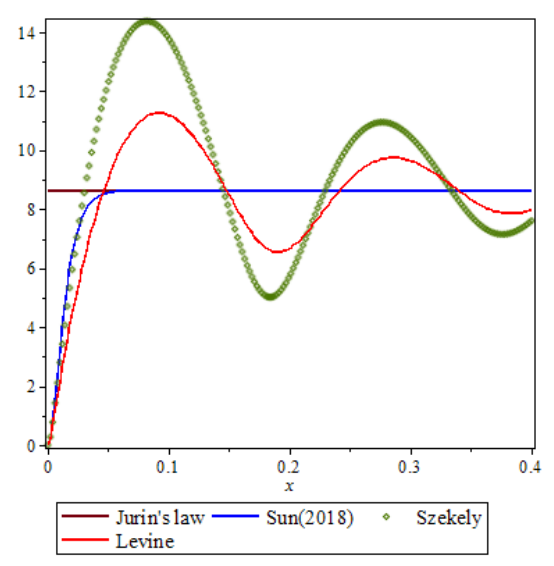

FIG. 2: Capillary height $h(t)$ profile for radius $a=0.5[\mathrm{~mm}]$ and comparisons.

Figure 3 shows that the profile of the capillary height $h(t)$ changes dramatically with the tube radius $a$. The phase transition from oscillating to monotonic rising happens at $a \approx 0.1 \mathrm{~mm}$, and the calculation indicates that to prevent oscillation, a thinner tube should be used.

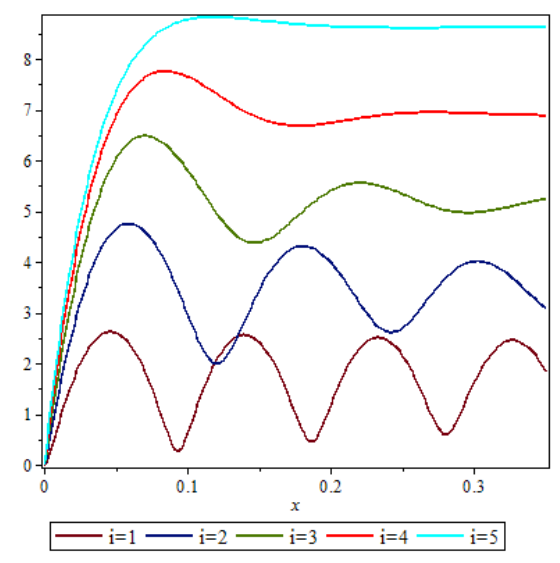

FIG. 3: Capillary height $h(t)$ profile for different tube radius $a=0.5 / i[\mathrm{~mm}]$, where $i=1,2,3,4$ and $i=5$. The capillary is oscillating at $a>0.1[\mathrm{~mm}]$ and monotonic at $a<0.1[\mathrm{~mm}]$.

The surface tension $\sigma$ is the source of the force that lifts the capillary, leading to the question of what happens if the value of $\sigma$ changes. Figure 4 shows that the capillary rise is strongly affected by the surface tension: the greater the surface tension, the higher the capillary rise.

If we only change the intake angle, Fig. 5 shows that the capillary height is affected slightly: the larger the angle, the smaller the capillary rise.

Similarly, Fig. 6 shows that the capillary height is affected slightly by changing the viscosity $\mu$.

The density plays a role in resisting capillary rise. Figure 7 shows that the capillary rise is strongly affected

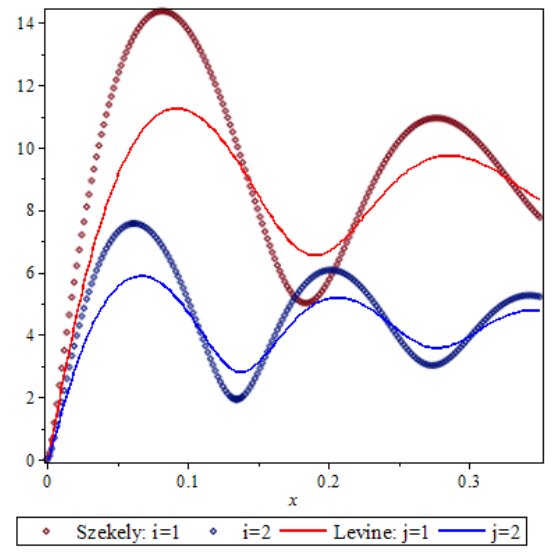

FIG. 4: Capillary height $h(t)$ profile for different surface tensor $\sigma=1.67 \times 10^{-2} / i$, where $i=1,2$.

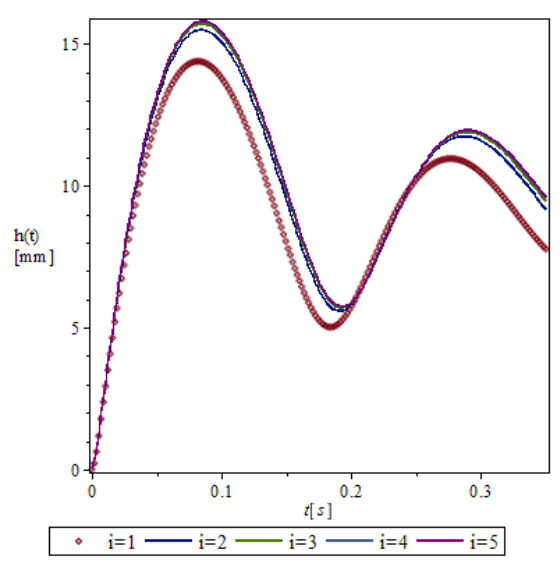

FIG. 5: Capillary height $h(t)$ profile for different angle $\theta=$ $26^{0} / i$, where $i=1,2$.

by the density: the greater the density, the lower the capillary rise.

The acceleration due to gravity is another factor that plays a role in resisting capillary rise, and Fig. 8 shows that the capillary rise is strongly affected by the acceleration due to gravity.

Figure 9 shows what the capillary rise would be on the Moon, there the acceleration due to gravity is $1.62 \mathrm{~m} / \mathrm{s}^{2}$, much less than that on Earth.

From the capillary-rise profiles for five different tube radii in Fig. 10, it is clear that the capillary rise would be much higher and with no oscillation; the rising is monotonic.

In the absence of gravity, Fig. 11 shows that the capillary rise would be even greater than that on the Moon.

In Fig. 12, comparison with Stange's experiments and modelling. 


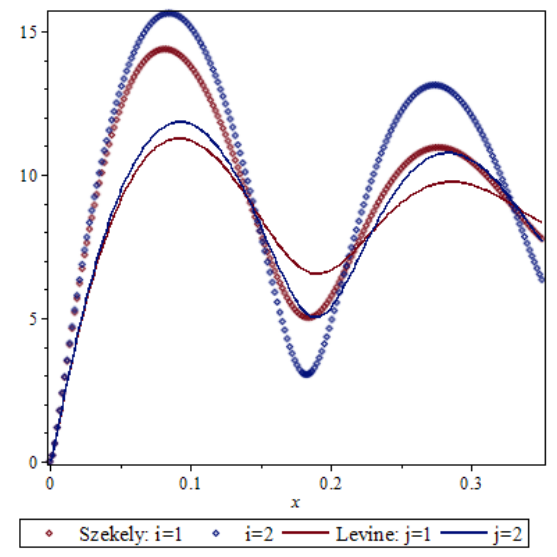

FIG. 6: Capillary height $h(t)$ profile for different viscosity $\mu=2.2 \times 10^{-4} / i$, where $i=1,2,3,4$ and $i=5$.

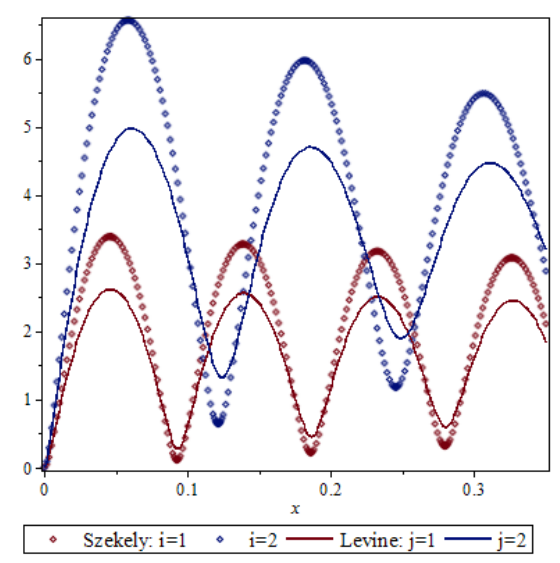

FIG. 7: Capillary height $h(t)$ profile for different density $\rho=$ $710 / i$, where $i=1,2,3,4$ and $i=5$.

\section{PHASE TRANSITION BETWEEN OSCILLATING AND MONOTONIC RISING}

Regarding the phase transition between oscillating and monotonic rising, there is no consensus on the critical tube radius. Assume oscillating solution $h(t)=H+y(t)$, and omit the 2nd order terms such as $y \ddot{y},(\dot{y})^{2}$ and $y \dot{y}$, we have an equation for $y(t): \ddot{y}+B \dot{y}+C y=0$, where $B=\frac{8 \mu}{\rho a} \frac{H}{H+\frac{7}{6} a}, C=\frac{g}{H+\frac{7}{6} a}$ and $H=\frac{2 \sigma}{\rho a g} \cos \theta$. The solution is $y(t)=e^{-\frac{1}{2} B t}\left(c_{1} \sinh \alpha t+c_{2} \cosh \alpha t\right)$, where $\alpha=\frac{1}{2} \sqrt{B^{2}-4 C}$ and $c_{1}, c_{2}$ are the constant of integration. Clearly, if $B^{2}-4 C<0$, the capillary rise oscillation and monotonic otherwise, hence $B^{2}-4 C=0$ gives a linear approximation gives the critical tube radius as

$$
a_{\text {Linear }} \approx\left(32 \frac{\sigma \mu^{2}}{\rho g^{2}} \cos \theta\right)^{1 / 3} .
$$

For the capillary with the data listed in Table I, the critical radius is predicted as $a \approx 0.07 \mathrm{~mm}$, which unfortunately contradicts the numerically predicted critical

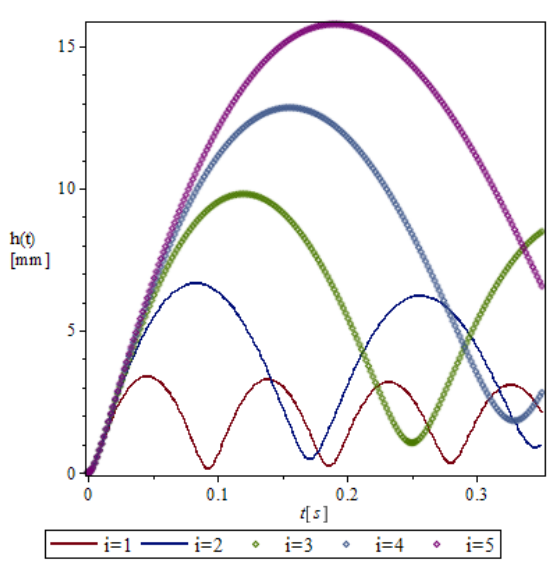

FIG. 8: Capillary height $h(t)$ profile for different gravity $g=$ $g / i$, where $i=1,2,3,4$ and $i=5$.

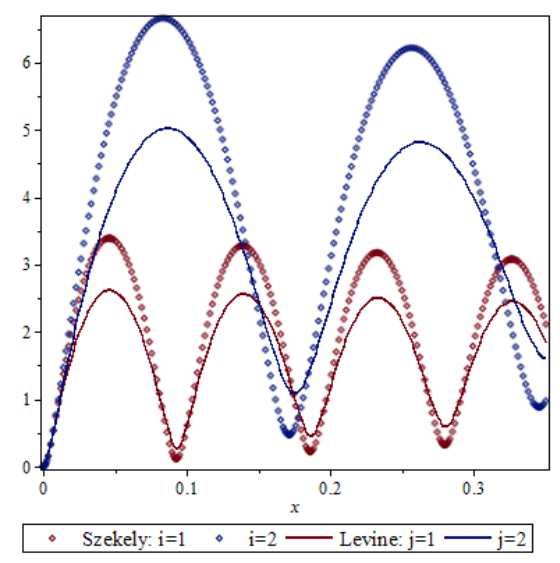

FIG. 9: Capillary height $h(t)$ profile: on moon with gravity $g=1.62\left[\mathrm{~m} / \mathrm{s}^{2}\right]$

value of $a \approx 0.2 \mathrm{~mm}$ as shown in Fig. 3 .

Therefore, the criterion for the phase transition from oscillating to monotonic rising remains unresolved. For the time being, from Buckingham's $\Pi$ theorem based on $a=f(\sigma, \mu, \rho, g, \theta)$. However, because $\sigma, \mu, \rho$, and $g$ are physical constants, only two dimensionless parameters can be produced, leading to a critical radius of $a=f\left(\frac{\sigma \mu^{2}}{\rho g^{2}}\right) \cos \theta$. Data fitting allows us to propose the phase-transition criterion as

$$
a_{c r} \approx \frac{1}{2}\left(\frac{\sigma \mu^{2}}{\rho g^{2}}\right)^{0.26} \cos \theta
$$

This approximation reveals that the critical radius of capillary tube is decreasing with the liquid density and gravity, and increasing with liquid surface tension and viscosity otherwise. 


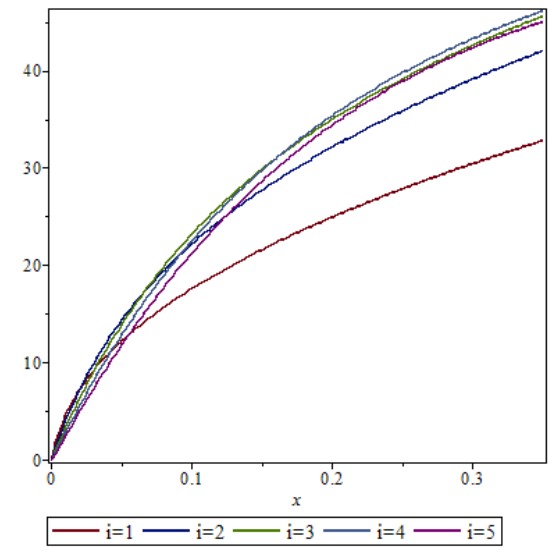

FIG. 10: Capillary height $h(t)$ profile: on moon with gravity $g=1.62\left[\mathrm{~m} / \mathrm{s}^{2}\right]$, numerical results for different radius $a=$ $0.1 i[\mathrm{~mm}]$

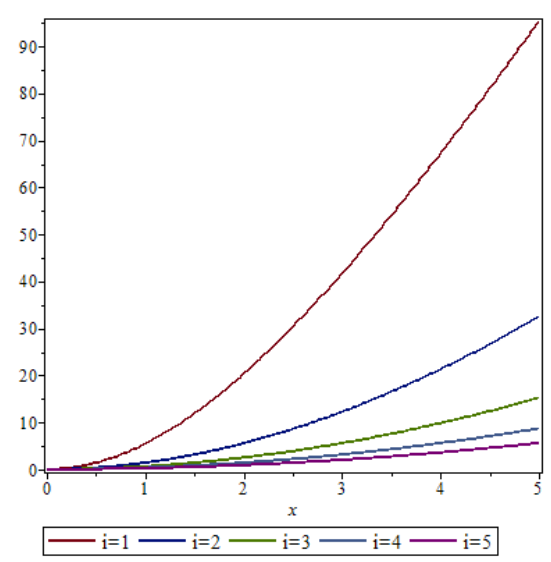

FIG. 11: Capillary height $h(t)$ profile: zero gravity; numerical results for different radius $a=0.1 i[\mathrm{~mm}]$

\section{DYNAMICS PHASE DIAGRAM}

Finally, we consider the phase diagram of capillary rise in Fig. 13. When the tube radius exceeds the critical radius (i.e., $a>a_{c r}$ ), because the capillary is damped by factors such as viscosity and gravity, the capillary rise loses energy during each oscillation cycle. As the amplitudes of the velocity and position decrease, the phase diagram spirals inwards. In classical mechanics, this is described as an "attractor" and shows that the system is trapped in a potential well from which it cannot escape. However, for monotonic rising with $a<a_{c r}$, the phase trajectory decreases exponentially to somewhere other than the attractor.

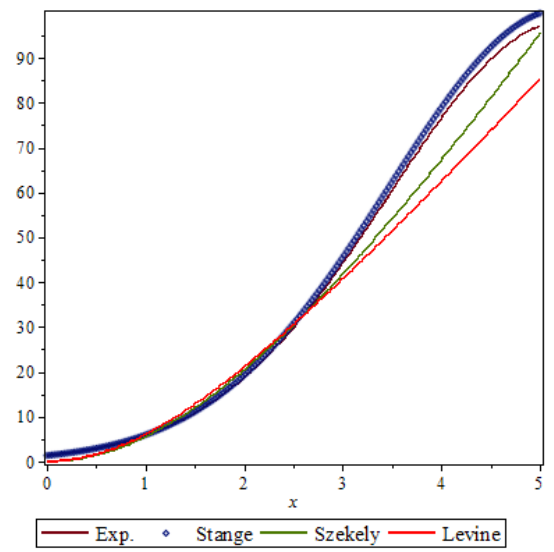

FIG. 12: Capillary height $h(t)$ profile comparison with Stange [12] in the case of no gravity. The minor misalignment after 3 seconds is because that Stange used different mathematical equation.

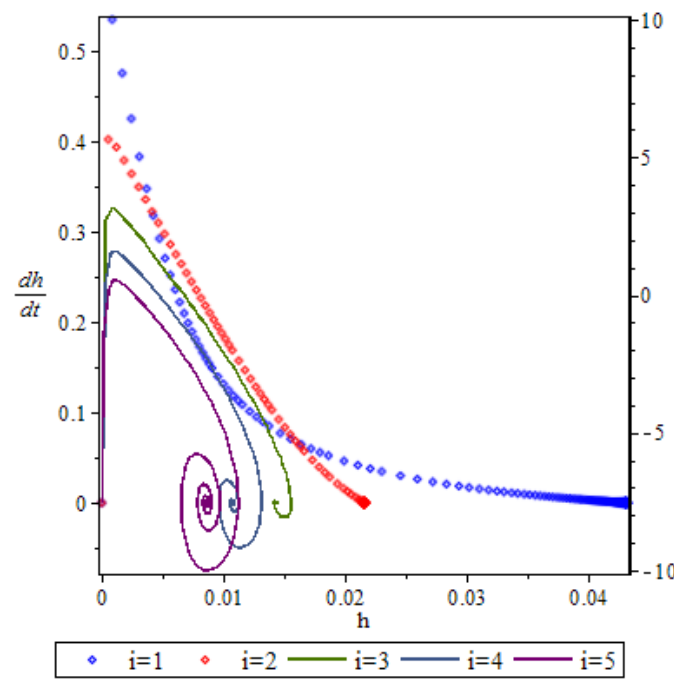

FIG. 13: Phase-space profile for different tube radius $a=$ $0.1 i[\mathrm{~mm}]$, where $i=1,2,3,4$ and $i=5$. The phase space diagram spirals inwards to a point "attractor" when tube radius $a>0.1[\mathrm{~mm}]$ and open with monotonic region at $a<0.1[\mathrm{~mm}]$. The capillary oscillation has attractor, while monotonic rise has no attractor.

\section{CONCLUSION}

The present results highlight how and when capillary dynamics are either oscillation or monotonic rise. This study has confirmed that the capillary height rising is strongly influenced by the tube radius, which is a dominate parameter. The critical radius is proposed by dimensional analysis and data fitting. The phase diagram of the capillary dynamics reveals that oscillation and monotonic rise have different attractors. 


\section{Appendix}

The Maple code for the Szekely's capillary dynamics equation:

$$
\left(h+\frac{7}{6} a\right) \frac{d^{2} h}{d t^{2}}+\frac{1}{2}\left(\frac{d h}{d t}\right)^{2}+\frac{8 \mu}{\rho a^{2}} h \frac{d h}{d t}+g h=\frac{2 \sigma}{\rho a} \cos \theta
$$

with initial height and velocity boundary conditions of $h(0)=0, \dot{h}(0)=0$.

for $\mathrm{i}$ from 1 to 5 do:

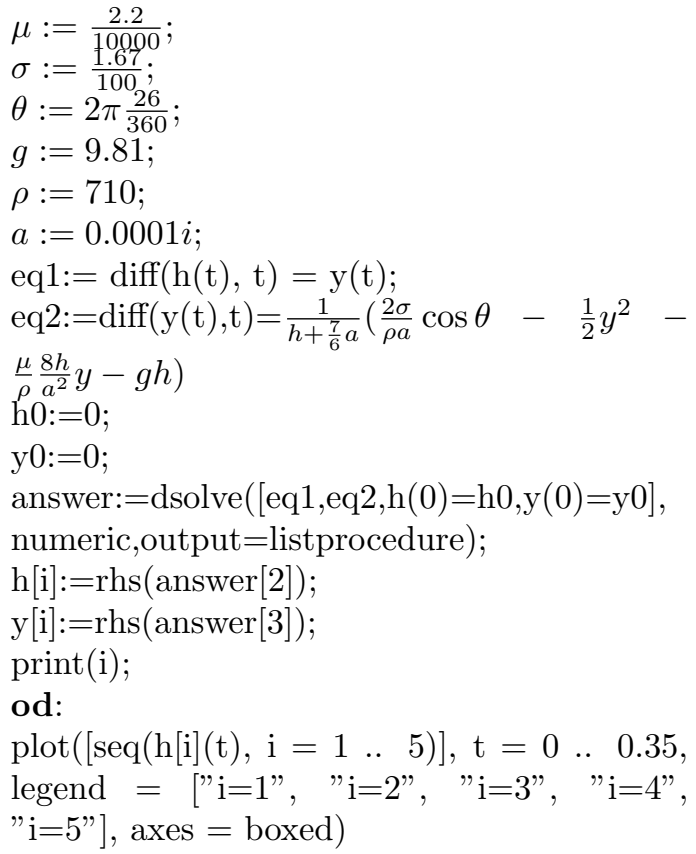

Availability of data: The data that support the findings of this study are available from the corresponding author upon reasonable request.

Conflict of interests: The authors declare that they have no known competing financial interests or personal relationships that could have appeared to influence the work reported in this paper

Bo-Hua Sun: Conceptualization, Methodology, Formulations, Formal analysis, Funding acquisition, Investigation, Writing- Original draft preparation, WritingReviewing and Editing and all relevant works.

[1] R. Lucas. Ueber das Zeitgesetz des kapillaren Aufstiegs von Flüssigkeiten. Kolloid-Zeitschrift, 23:15-22 (1918).
[2] E.W. Washburn. The dynamics of capillary flow. Physical Review, 17(3):273-283 (1921).

[3] E.K. Rideal. On the flow of liquids under capillary pressure. Philos. Mag. Ser. 6, 44:1152-1159 (1922).

[4] C.H. Bosanquet. On the flow of liquids into capillary tubes. Philos. Mag. Ser. 6, 45:525-531 (1923).

[5] W.E. Brittin. Liquid rise in a capillary tube, J. Applied Physics, 17:37-45 (1946).

[6] S. Newman. Kinetics of wetting of surfaces by polymers - capillary flow, J. of Colloid and Interface Science, 26(2)209-213 (1968).

[7] J. Szekely, A.W. Neumann and Y.K. Chuang. The rate of capillary penetration and the applicability of the washburn equation. J. Colloid and Interface Science, 35, 273278 (1971).

[8] S. Levine, P. Reed, E. J. Watson, and G. Neale, A theory of the rate of rise of a liquid in a capillary, Colloid and Interface Science, edited by M. Kerker Academic, New York, 1976, Vol. III, $403-419$.

[9] M. Dreyer, A. Delgado and H.J. Rath. Fluid motion in capillary vanes under reduced gravity, Microgravity Science and Technology, 4:203-210 (1993).

[10] J. Fisher. Physics takes the biscuit, Nature, 397,469 (1999).

[11] B.V. Zhmud, F. Tiberg, K. Hallstensson. Dynamic$\mathrm{s}$ of capillary rise. J. Colloid and Interface Science, 228(2):263-269 (2000).

[12] M. Stange, M. E. Dreyer, and H. J. Rath, Capillary driven flow in circular cylindrical tubes, Phys.Fluids, 15,9:2587-2601 2003.

[13] P. G. de Gennes, F. Brochard-Wyart and D. Quéré. Capillarity and Wetting Phenomena: Drops, Bubbles, Pearls, Waves. (Springer, New York, 2003).

[14] Y. Xiao, F. Yang and R. Pitchumani, A generalized analysis of capillary flows in channels, J. Colloid and Interface Science, Vol. 298, pp: 880-888 (2006).

[15] N. Fries and M. Dreyer. An analytic solution of capillary rise restrained by gravity, 320, 1:259-263 (2008).

[16] Q.Z. Yuan and Y.P. Zhao. Precursor film in dynamic wetting, electrowetting and electro-elasto-capillarity. Physical Review Letters, 104: 246101 (2010).

[17] Y.P. Zhao. Physical Mechanics of Surface and Interface (in Chinese)(Science Press, Beijing, 2012).

[18] J.W.M. Bush. Interfacial Phenomena, MIT Open Course (2013).

[19] B.H. Sun, Singularity-free approximate analytical solution of capillary rise dynamics, Sci. China-Phys. Mech. Astron. 61, 084721 (2018), https://doi.org/10.1007/s11433-018-9247-1

[20] X.X. Zhong, B.H. Sun and S.J. Liao, Analytic solutions of the rise dynamics of liquid in a vertical cylindrical capillary, European Journal of Mechanics / B Fluids 78 (2019) 1-10, https://doi.org/10.1016/j.euromechflu.2019.05.011

[21] Maple https://www.maplesoft.com/ 